Research paper

\title{
A GBM-like V-ATPase signature directs cell-cell tumor signaling and reprogramming via large oncosomes
}

\author{
Irene Bertolini ${ }^{\mathrm{a}, 1}$, Andrea Terrasi ${ }^{\mathrm{a}, 1}$, Cristina Martelli $^{\mathrm{b}}$, Gabriella Gaudioso ${ }^{\mathrm{a}}$, Andrea Di Cristofori ${ }^{\mathrm{c}}$, \\ Alessandra Maria Storaci ${ }^{\mathrm{a}, \mathrm{b}}$, Miriam Formica ${ }^{\mathrm{d}}$, Paola Braidotti ${ }^{\mathrm{e}}$, Katia Todoerti ${ }^{\mathrm{f}}$, Stefano Ferrero ${ }^{\mathrm{a}, \mathrm{g}}$, \\ Manuela Caroli ${ }^{\text {c }}$, Luisa Ottobrini ${ }^{\text {b }}$, Thomas Vaccari ${ }^{\mathrm{g}, *, 2}$, Valentina Vaira ${ }^{\mathrm{a}, \mathrm{b}, \mathrm{h}, * *, 2}$
}

a Division of Pathology, Fondazione IRCCS Ca' Granda Ospedale Maggiore Policlinico, Milan, Italy

b Department of Pathophysiology and Transplantation, Università degli Studi di Milano, Milan, Italy

' Division of Neurosurgery, Fondazione IRCCS Ca' Granda Ospedale Maggiore Policlinico, Milan, Italy

d Department of Biosciences, Universita' degli Studi di Milano, Milan, Italy

e Division of Pathology, San Paolo Hospital, Milan, Italy

${ }^{f}$ Department of Oncology and Hemato-oncology, University of Milan, Hematology Division, Fondazione IRCCS Ca' Granda Ospedale Maggiore Policlinico, Milan, Italy

g Department of Biomedical, Surgical and Dental Sciences, University of Milan, Milan, Italy

${ }^{\text {h }}$ Fondazione Istituto Nazionale Genetica Molecolare 'Romeo ed Enrica Invernizzi', Milan, Italy

\section{A R T I C L E I N F O}

\section{Article history:}

Received 23 November 2018

Received in revised form 24 January 2019

Accepted 25 January 2019

Available online xxxx

\section{Keywords:}

V-ATPase

Homeobox genes

Glioma stem cells

Large oncosome

\begin{abstract}
A B S T R A C T
Background: The V-ATPase proton pump controls acidification of intra and extra-cellular milieu in both physiological and pathological conditions. We previously showed that some V-ATPase subunits are enriched in glioma stem cells and in patients with poor survival. In this study, we investigated how expression of a GBM-like V-ATPase pump influences the non-neoplastic brain microenvironment.

Methods: Large oncosome (LO) vesicles were isolated from primary glioblastoma (GBM) neurospheres, or from patient sera, and co-cultured with primary neoplastic or non-neoplastic brain cells. LO transcript and protein contents were analyzed by qPCR, immunoblotting and immunogold staining. Activation of pathways in recipient cells was determined at gene and protein expression levels. V-ATPase activity was impaired by Bafilomycin A1 or gene silencing. Findings: GBM neurospheres influence their non-neoplastic microenvironment by delivering the V-ATPase subunit V1G1 and the homeobox genes HOXA7, HOXA10, and POU3F2 to recipient cells via LO. LOs reprogram recipient cells to proliferate, grow as spheres and to migrate. Moreover, LOs are particularly abundant in the circulation of GBM patients with short survival time. Finally, impairment of V-ATPase reduces LOs activity.

Interpretation: We identified a novel mechanism adopted by glioma stem cells to promote disease progression via LO-mediated reprogramming of their microenvironment. Our data provide preliminary evidence for future development of LO-based liquid biopsies and suggest a novel potential strategy to contrast glioma progression. Fund: This work was supported by Fondazione Cariplo (2014-1148 to VV) and by the Italian Minister of HealthRicerca Corrente program 2017 (to SF).
\end{abstract}

(C) 2019 Published by Elsevier B.V. This is an open access article under the CC BY-NC-ND license (http:// creativecommons.org/licenses/by-nc-nd/4.0/).

\section{Introduction}

Glioblastoma (GBM) is a very aggressive form of malignant brain tumor and one of the least treatable cancers. One of the most important enabling features of GBMs is the ability to subvert the normal tissue

\footnotetext{
DOI of original article: https://doi.org/10.1016/j.ebiom.2019.01.052.

* Correspondence to: Thomas Vaccari, via Celoria 26, 20133 Milan, Italy.

** Correspondence to: Valentina Vaira, via F. Sforza 35, 20122 Milan, Italy.

E-mail addresses: thomas.vaccari@unimi.it (T. Vaccari),valentina.vaira@unimi.it (V. Vaira).

1 Joint first authors.

2 Joint last authors.
}

environment to supply their growth needs [1]. In principle, a deep understanding of the means by which the tumor reprograms its surroundings could prompt development of new drugs or treatments to slow down, and eventually eradicate, the disease.

The ability of a tumor to modify its microenvironment is a major hallmark of malignant progression [2]. Instructing untransformed cells to take part in development of the tumor organ requires highly regulated cell-cell and cell-stroma communication. Most cells are known to secrete a number of extracellular vesicles (EVs) that carry signaling molecules within the nervous system [3]. EVs secreted by tumors are known to carry RNAs and signaling molecules that alter the fate of receiving cells [4,5]. An emerging mode of delivery of extracellular signals 


\section{Research in context}

\section{Evidence before this study}

The V-ATPase proton pump has been involved in cancer stem cells maintenance and V-ATPase-high glioblastomas express higher levels of homeobox genes. Moreover, the V-ATPase has been involved in synaptic vesicles uptake/release and in the modulation of the microenvironment.

\section{Added value of this study}

We show that GBM neurospheres with high levels of V-ATPase expression secrete large oncosomes (LOs), a type of extracellular vesicle loaded with oncogenic cargoes. These LOs carry homeobox factors and V-ATPase subunit $\mathrm{G} 1$ and are able to reprogram the brain microenvironment toward a pro-tumorigenic state. Finally, LOs are also found in the circulation of glioma patients and are poor prognostic factors.

\section{Implications of all the available evidence}

A role for $\mathrm{V}$-ATPase in the modification of the surrounding milieu is emerging. These data identify a global cell fate change that underlies glioma aggressiveness and suggest new entry points for glioma patients' follow-up and therapy.

by cancer cells is that mediated by large oncosomes (LOs). LOs are formed when a large portion of cellular membranes is shed from the cell surface during blebbing events [6,7]. GBM secrete a number of vesicles carrying a large variety of signals in the form of proteins and RNAs that can also be recovered from body fluids of patients [8-10]. However, how EVs, and in particular LOs, reprogram their surrounding microenvironment is poorly known. Importantly, it has been shown that the genetic and phenotypic intratumor heterogeneity is also reflected in the EVs' repertoire [11]. Indeed EVs might contribute to glioma cell plasticity and to influence functional integration of glioma stem cells (GSC) with other cancer cell populations. In this context, the understanding of GSC vesiculome could provide novel insights into mechanisms of disease progression and resistance to therapy.

The vacuolar ATPase (V-ATPase) is a proton pump involved in a number of acidification-dependent processes in the cell in physiological as well as pathological contexts [12]. In a recent study, we have determined that V1G1, a subunit of the V-ATPase that is highly expressed in GBM, plays a functional role in GSC and can be used as an accurate prognostic marker [13]. In the accompanying study published in this issue, we show that differences between aggressive GBMs and lower grades gliomas include not only V1G1 but also a number of other V-ATPase subunits. We also reveal the existence of a GBM transcriptional profile that, in addition to many V-ATPase subunits, comprises also high expression of Hox genes resembling that of undifferentiated embryonic cells (Terrasi et al., this issue) [36]. However, whether such genes are also able to influence the tumor surrounding milieu possibly through EVs is not known.

Therefore, we analyzed GSC derived LOs and their functional effects in neoplastic and non-neoplastic brain cells to get insights into a novel $\mathrm{V}$-ATPase role in gliomagenesis.

\section{Materials and methods}

\subsection{Primary, patients-derived or commercial cell cultures}

GBM neurospheres (NS, $n=33$ ) were obtained from fresh tumor tissue of GBM patients as previously described [13]. For LO co-culture experiments, recipient primary cells were isolated directly from patients' non-neoplastic (margins, $\mathrm{n}=22$ ) taken outside the tumor area, or tumor $(\mathrm{n}=18$ ) tissues. To confirm tissues histology, a portion of each sample was snap-frozen and a section was stained with hematoxylin and eosin (H\&E). All patients were enrolled at the Division of Neurosurgery and glioma was diagnosed at the Division of Pathology of the Fondazione IRCCS Ca' Granda Ospedale Maggiore Policlinico (Milan, Italy). This study was approved by a local Ethic Committee (IRB\#275/2013) and all patients signed an informed consent.

All cases were reviewed and graded by senior pathologist (SF) according to the new classification of the World Health Organization (WHO [14];). To obtain single cell suspensions, tissues underwent enzymatic and mechanical dissociation digestion in serum-free media using the Tumor Dissociation kit (Miltenyi). Then, cells suspensions were washed twice with HBSS (Gibco-Invitrogen, Thermo Fisher Scientific, Waltham, MA USA) and seeded in NeuroCult (NC) media supplemented with growth factors (Proliferation Supplement, bFGF and EGF, all from Voden). Margin cultures were immunoprofiled by immunofluorescence or FACS using the following antibodies: Nestin, Tuji, GFAP, CD11b, 04, Olig2, CD31 and Vimentin. For FACS analysis, cells were fixed in PFA $2 \%$ for $10 \mathrm{~min}$, permeabilized in Triton $0.1 \%$ for $10 \mathrm{~min}$ and stained with primary $(1: 100 / 1 \mathrm{~h})$ and secondary antibody $(1: 1000 / 30 \mathrm{~min})$ at room temperature (RT). Unstained cells were used as negative control, whereas cells incubated with immunoglobulin of the same isotype of the primary antibody were used as isotype control.

LN229 cells were purchased from ATCC (Manassas, VA, USA) and maintained in RPMI media supplemented with $10 \%$ exosome-free FBS (Exo-FBS, System Biosciences).

\subsection{Immunohistochemistry (IHC) in GBM patients' derived orthotopic} mouse model xenograft or in human glioma tissues

Brains from mice inoculated with vehicle (controls) or primary GBM NS characterized by low or high V-ATPase G1 expression were harvested at sacrifice and formalin-fixed and paraffin embedded (FFPE) as described in the companion study (Terrasi et al., this issue) [36]. Then, hematoxylin and eosin (H\&E) staining was performed to confirm tissue morphology followed by immunohistochemistry for HOXA10 (TA590263, Origen), STEM121 (Y40410, Takara Bio Europe) and POU3F2 (12137, Cell Signaling). Human tissues were already FFPE blocks. IHC for V-ATPase G1 (16143-1-AP, Proteintech), Nestin (MAB1259, R\&D Systems), HOXA10, or POU3F2 was performed using a Ventana Benchmark instrument and the Ultraview DAB or Red Detection kits as described [13]. For murine samples, slides were preincubated with the Rodent Block $M$ reagent (Biocare Medical), to block endogenous mouse IgG and non-specific background, before primary antibodies. Percentage of positive tumor cells in PDTX tumors was analyzed using Aperio Digital Pathology slide scanner (Leica Biosystems, Milan, Italy) and the nuclear algorithm implemented in ImageScope software (Leica Biosystems) as described [15].

\subsection{LO isolation from NS media or patients' sera}

LO were isolated from supernatant of NS $(\mathrm{n}=12)$ at basal condition (after $24 \mathrm{~h}$ of NS culture with fresh media), after $10 \mathrm{nM}$ BafA1 treatment of NS for $24 \mathrm{~h}$, or after siRNA-mediated knockdown of V-ATPase G1 in NS as described [7]. Briefly, cells and debris were removed by centrifugation at $250 \mathrm{rcf}$ for $5 \mathrm{~min}$ followed by $1.000 \mathrm{rcf}$ for $10 \mathrm{~min}$ at $4{ }^{\circ} \mathrm{C}$. Finally, supernatants were collected and concentrated using Amicon Ultra centrifugal filter tubes (Millipore). Supernatants were then centrifuged at $10.000 \mathrm{rcf}$ for $30 \mathrm{~min}$ at $4{ }^{\circ} \mathrm{C}$. Pellets containing LO were then washed with $0.2 \mu \mathrm{m}$-filtered PBS, resuspended in filtered PBS and stored at $-80{ }^{\circ} \mathrm{C}$ before using for functional experiments or RNA/protein extraction. Vesicles concentration and size distribution was analyzed using Nanosight NS300 (Malvern, Instruments Ltd. Worcestershire, UK). LO were stored as single aliquots of $20 \mu \mathrm{l}$ and, for functional 
experiments, vesicles were provided to recipient cells at a final concentration of $3 \times 10^{8} \mathrm{LO} / \mathrm{ml}$ (which corresponded to a single $20 \mu \mathrm{l}$-aliquot). For controls (mock samples), fresh NC medium was processed as culture media and stored at $-80^{\circ} \mathrm{C}$. Fresh patients' sera were processed immediately after blood withdrawal as previously reported [16]. Briefly, sera were centrifuged at $700 \mathrm{rcf}$ for $15 \mathrm{~min}$ at $4{ }^{\circ} \mathrm{C}$, after which LO isolation was performed using $500 \mu \mathrm{l}$ of the supernatant and processed as described for cell culture media.

\subsection{Analysis of LO evagination from NS by confocal microscopy}

To investigate LO production from live NS, cells were incubated for 30 min at $37{ }^{\circ} \mathrm{C}$ with FM 1-43 FX at $40 \mathrm{uM}$ (ThermoFisher Scientific). After two washes in PBS, NS were seeded on glass bottom well (MatTek) and followed live for 20 min using the gas/humidification chamber of the confocal microscope (TSC5). Images were captured every 5 min and LO (dimension between 1 and $5 \mu \mathrm{m}$ ) were identified as evagination of the plasma membrane.

\subsection{Flow cytometry of LO}

LO were stained for $20 \mathrm{~min}$ at $37^{\circ} \mathrm{C}$ with CellTrace Violet $(5 \mu \mathrm{M}$, intact membrane staining) and SYTO RNASelect (10 $\mu \mathrm{M}$, RNA staining), washed twice with filtered PBS and visualized using a FACSCanto II instrument (BD Biosciences). As negative control (mock samples), we used LO isolated from empty NeuroCult media. FlowJo V.10.1 software (BD Biosciences) was used to analyze data.

\subsection{LO internalization}

For internalization assays, LO or control were stained with PKH26 dye (Sigma Aldrich) or with FM 1-43 FX at 40 uM (ThermoFisher Scientific). Briefly, dyes were added to LO pellets for $5 \mathrm{~min}$ at RT and the staining was blocked with $10 \%$ BSA in filtered PBS. LO preparations were then washed three times and pelleted at $10.000 \mathrm{rcf}$ for $30 \mathrm{~min}$. Recipient cells (primary GBM or margin cells) were seeded on 96-wells glass bottom plates (MatTek Ashland, MA) at 80\% confluence and stained with the vital dyes CellTrace Violet ( $5 \mu \mathrm{M}$, cytoplasm staining) and CellsMask (1:1000, cytoplasm and plasma membrane staining) for $30 \mathrm{~min}$ at $37^{\circ} \mathrm{C}$ in PBS. Then, cells were rinsed twice with filtered PBS and fresh medium was added. After 24 h, one aliquot of PKH26stained LO or vehicle was added to recipient cells. LO internalization was evaluated after 6 and $24 \mathrm{~h}$ of co-culture in live cells using live confocal microscopy and a 63X objective. Leica software tools line profile intensity - sort ROI (Leica Microsystems LAS AF 2.6.0.7266) were used to discriminate LO internalized in recipient cells from LO bound to the plasma membrane. In particular, for each image the intensity of the three dyes profiles was investigated in $3 \mathrm{z}$-stacks corresponding to the cell top, middle and bottom.

\subsection{Functional experiments}

For evaluation of cell growth after LO co-culture, recipient cells were seeded in 24 multi-wells plates and let reach $60 \%$ of confluence. Then cultures were stained with CellTrace Violet $\left(5 \mu \mathrm{M}\right.$ for $20 \mathrm{~min}$ at $37^{\circ} \mathrm{C}$ ) and one aliquot of LO or vehicle (mock samples) was added per well. The percentage of divided cells was evaluated as the decrease in CellTrace Violet fluorescence intensity, whereas the number of cell divisions was analyzed using the FlowJo software tool "proliferation". Both analyses were performed at 3,10 and 17 days after co-culture using FACS and analyzed by FlowJo V.10.1 software. To assess the effect of LO on neurospheres generation, primary GBM-derived cultures were seeded in 48-wells plates in NeuroCult media and observed for 2 weeks. If the culture did not form NS, one LO or vehicle aliquot was added per well. After $24 \mathrm{~h}$ supplements were removed and sphere formation was monitored at 3,6 and 90 days as previously described [13].
For invasion assays, NS with low V-ATPase G1 level ( $\mathrm{LO}^{\text {Low }}$ ) were preincubated with LO from NS with high or low V-ATPase G1 expression ( $\mathrm{LO}^{\mathrm{High}}$ or $\mathrm{LO}^{\mathrm{Low}}$ ) and then embedded in a collagen matrix (C4243; Sigma Aldrich). Cell spreading through the matrix was monitored up to 3 days and images were captured every $24 \mathrm{~h}$ using a time-lapse microscope (Eclipse Ti-E, Nikon Instruments; Florence, Italy). The radius of invasion was calculated as the distance of invaded cells from the edge of the NS using Volocity software (PerkinElmer, Waltham, MA, USA). NS clonogenicity assay was previously described [13].

\subsection{Electron microscopy (EM) and immunogold staining}

For ultrastructural morphology examination, spheroids were fixed in $2.5 \%$ glutaraldehyde, embedded in $2 \%$ agar solution, post-fixed in $1 \%$ osmium tetroxide in phosphate buffer, dehydrated and embedded in epoxy resin. Ultra-thin sections $(50-60 \mathrm{~nm}$ ) were counterstained with uranyl acetate, lead citrate and observed in a Jeol JEM 1010 (Tokyo, Japan) transmission electron microscope. Images were then captured using a FEI Tecnai G2 20 Transmission Electron Microscope. To identify multivesicular bodies, intracellular images were captured at 1840X magnification, whereas to evaluate extracellular vesicles (exosomes, ectosomes and large oncosomes) presence and production, images of the extracellular milieu were captured at 5000X magnification. Quantification of EVs diameter in TEM images was performed using Volocity (PerkinElmer Inc., Waltham, MA) software. Briefly, EVs were manually selected with a circular ROI as objects and their diameter was measured to identify LO ( $\geq 1 \mu \mathrm{m})$. Then LO number per area of extracellular space was calculated for each NS type. Isolated LO were prepared for EM following the protocol of Lobb et al. [17] Briefly, $7 \mu$ l of samples were absorbed on glow-discharged carbon-coated formvar nickel grids for $2 \mathrm{~min}$. The drops were then blotted with filter paper and negatively stained with $2 \%$ uranyl acetate $(5 \mu \mathrm{l})$ in aqueous suspension for $2 \mathrm{~min}$. Excess of uranyl was removed by touching the grid with filter paper and the grid was dried at RT. Grids were examined at Zeiss LEO 912ab electron microscope at $80 \mathrm{kv}$. To evaluate the presence of V-ATPase G1 or of HOXA10 in LO, immunogold labeling was carried out. Few microliters of the sample were absorbed on glow-discharged carbon-coated formvar nickel grids, blocked in 1\% BSA in PBS and incubated with primary antibody (1:100). After several washes in 0.1\% BSA in PBS, samples were incubated for $30 \mathrm{~min}$ with goat anti-rabbit IgG conjugated with $10 \mathrm{~nm}$ colloidal gold particles (BBInternational), fixed with $1 \%$ glutaraldehyde, stained with $2 \%$ uranyl acetate, and air-dried. Grids were observed with a Zeiss LEO 912 transmission electron microscope. Images were acquired by a $2 \mathrm{k} \times 2 \mathrm{k}$ bottom-mounted slow-scan Proscan camera controlled by EsivisionPro 3.2 software. Images were captured at 12.500 and $20.000 \mathrm{X}$ magnification.

\subsection{RNA purification and genes expression analyses}

Total RNA was purified from cell cultures and large oncosomes (LO) using Master Pure RNA purification kit (Epicentre Biotechnologies, Illumina; Madison, WI, USA) followed by DNA digestion with DNase I, Amplification Grade (Thermo Fisher Scientific). In co-culture experiments, mRNA was extracted after 3 or 6 and 90 days after LO supplementation of margins or GBM tumor cells cultures. Then, $300 \mathrm{ng}$ of DNA-free total RNA from cell cultures were reverse transcribed using the SuperScript II Reverse Transcriptase and random hexamers (both from Thermo Fisher Scientific) in $20 \mu \mathrm{l}$ or reaction mix. For LO, $100 \mathrm{ng}$ of DNA-free total RNA were retrotranscribed and preamplified using a custom PreAmp primers pool, prepared diluting TaqMan assays (listed in Supplementary Table 8) in 1X TE buffer to a final concentration of 0.2.Targets gene expression was quantified by qPCR using TaqMan assays together with the reference genes $\beta 2$ microglobulin and/or $18 \mathrm{~S}$ RNA. Genes expression was evaluated by quantitative PCR (qPCR) and target genes were relatively quantified on the reference transcripts average using the $2^{\wedge}(-$ Delta $\mathrm{Ct}$ ) formula. Then, targets relative quantities 
(RQ) were median normalized and log2-transformed. For digital PCR (dPCR) analysis, we used the QuantStudio Digital PCR Kit (Thermo Fisher scientific) and the absolute amount (copies per $\mu \mathrm{l}$ ) of POU3F2 transcript in LO from patients' sera or NS media was quantified from $25 \mathrm{ng}$ of the previously obtained cDNA. For transcriptomic analysis of LO-or mock-cocultures ( $\mathrm{n}=2$ pairs), RNA was purified as before and RNA integrity was verified by Agilent Bioanalyzer (Agilent Technologies). Then samples were processed on a GeneChip Human Gene 2.0 ST Array (Affymetrix, part of Thermo Fisher Scientifics), as previously described [18]. Raw data were preprocessed and normalized using the Robust Multi-array Average (RMA) method implemented in the R package "Affy". Expression data were then analyzed using Gene Set Enrichment Analysis (GSEA) module available within Genepattern (http:// software.broadinstitute.org/cancer/software/genepattern/modules/ docs/GSEA/14) using the "gene_set" as permutation type and all other default parameters. Raw and processed data is deposited in GEO public database (accession number: GSE120793).

\subsection{0. siRNA transfections}

GBM primary cultures were transiently transfected in Optimem media using Lipofectamine 3000 (both from Invitrogen, Thermo Fisher Scientific) and $100 \mathrm{pM}$ of specific or non-targeting control siRNAs or esiRNA (all from Sigma Aldrich, St. Louis, MO, USA). The siRNA SASI_Hs02_00338522 was used to silence V-ATPase G1 transcript as described [13]. After $5 \mathrm{~h}$, transfection mixture was replaced with standard culturing media.

\subsubsection{Antibodies and reagents}

Lysotracker, CellTrace Violet, FM lipophilic styryl (FM 1-43FX) and SYTO RNASelect dyes were from Life Technologies (Thermo Fisher Scientific). Acridine Orange was from ImmunoChemistry Technologies (Bloomington, MN, USA). PropidiumIodure (PI) and Annexin V were from BD Bioscience (FITC-Annexin V Apoptosis Detection Kit). Bafilomycin A1 (BafA1; sc-201550) was from Santa Cruz Biotechnologies whereas NH4Cl (254134) was from Sigma Aldrich.

The following primary antibodies were used for immunofluorescence, immunoblotting (IB) and flow cytometry (FACS) assays: VATPase G1 (16143-1-AP, Proteintech), Vinculin (V9131, Sigma Aldrich), Tsg101 (14497-1-AP, Proteintech), Ago2 (10686-1-AP, Proteintech), Clathrin (ab23440, Abcam), CD63 (sc-15363, Santa Cruz), CD9 (IB: 10626D, Thermo Fisher; FACS: 130-103-988, Miltenyi), CD81 (130107-982, Miltenyi), Calnexin (ab31290, Abcam), Nestin (MAB 1259, R\&D), Tuji (T3952, Sigma Aldrich), GFAP (G9269, Sigma Aldrich), CD11b (20991-1-AP, Proteintech), 04 (07139, Sigma Aldrich), Olig2 (AV32753, Sigma Aldrich), CD31 (ab28364, Abcam), Vimentin (130106-369, Miltenyi), POU3F2 (12137, Cell Signaling), HOXA10 (TA590263, Origen), HOXA7 (ab211521, Abcam), V-ATPase G2 (25316-1-AP, Proteintech), ARF6 (6ARF01, ThermoFisher Scientific), and GRP78 (3177, Cell Signaling).

\subsection{Immunofluorescence}

For immunofluorescence experiments, monolayer cultures were grown on cover-glasses whereas NS were cytospinned on charged slides for $3 \mathrm{~min}$ at $900 \mathrm{rpm}$ (Thermo Scientific, Waltham, MA, USA). Cells were fixed in PFA 4\% for 15 (monolayer cultures) or $30 \mathrm{~min}$ (NS), quenched for green auto-fluorescence using glycine $20 \mathrm{mM}$ for $20 \mathrm{~min}$ and then permeabilized with Triton $0.5 \%$ for $30 \mathrm{~min}$ at room temperature. Then, samples were saturated with BSA $10 \%$ for $1 \mathrm{~h}$ at RT. Primary antibodies (1:100 in PBS-BSA $10 \%)$ were incubated overnight at $4{ }^{\circ} \mathrm{C}$ whereas secondary antibodies (1:1000) were incubated for $1 \mathrm{~h}$ at room temperature. Finally, nuclei were stained with Hoechst 3342 (Cell Signaling) for $5 \mathrm{~min}$ at RT and slides were mounted using the Prolong reagent (ThemoFisher Scientific). For negative controls, one slide per target protein was incubated with all reagents but the primary antibody. Confocal images were generated with a Leica TCS SP5 Confocal microscope (Leica Microsystems, Milan, Italy) with a magnification of $40 X$ or $63 X$, and zstack images were captured every $0.46 \mu \mathrm{m}$. Fluorescence quantification was calculated on full cell stacks after setting the threshold on control samples, and the mean intensity of the fluorochrome was calculated using ImageJ software.

\subsection{Immunoblotting}

Cells and LO protein extracts were prepared in RIPA buffer supplemented with phosphatase and protease inhibitors (Roche). For LO preparations, 3\% SDS was added as pretreatment. After lysis, samples were sonicated five times on ice (Bioruptor Plus sonication device, Diagenode), pelleted at $15.000 \mathrm{rcf}$ to remove debris and quantified using microBCA kit (Thermo Fisher Scientific). Proteins ( $40 \mu \mathrm{g}$ ) were boiled for $5 \mathrm{~min}$ at $95^{\circ}$ (in non-reducing condition for CD63 detection), resolved by SDS-PAGE, transferred to nitrocellulose membrane, blocked in 5\% not-fat powdered milk in PBS-T (1\% Tween-20) and probed with antibodies. Proteins bands were then visualized using the Amersham ECL Prime Western Blotting Detection Reagent (GE Healthcare) and the LAS-3000 imager (Fujifilm).

\subsection{Statistical analyses}

Data from functional experiments were imported in GraphPad Prism software (La Jolla, CA, USA) and analyzed using the non-parametric Mann-Whitney U and Kruskal-Wallis tests for two-sample or more than two-sample comparisons, respectively. Data are presented as mean \pm SEM and experiments were performed at least three times and in technical duplicate unless otherwise specified. In particular, experiments involving patients' derived NS were performed with five $\mathrm{V} 1 \mathrm{G} 1^{\mathrm{High}}$ - and five V1G1 ${ }^{\text {Low }}$ - NS in triplicate. LO were isolated from 12 NS, equally distributed for V-ATPase G1 expression for LO co-cultures with GBM recipient cells ( $\mathrm{n}=10$ ), we performed biological triplicate for each patients' sample, whereas non-neoplastic margin cultures (n $=20$ ) were analyzed using a single technical replicate because of the limited material available. Categorical data were analyzed using chi square test. P values $<.05$ were considered statistically significant if not otherwise specified.

\section{Results}

3.1. GBM with elevated V-ATPase G1 expression display upregulation of homeobox genes in vivo

In the accompanying paper we determined that GBM neurospheres with elevated V-ATPase G1 (V1G1 ${ }^{\text {High }} \mathrm{NS}$ ) were also enriched for the homeobox genes HOXA7, HOXA10 and POU3F2 (Terrasi et al. in this issue) $[13,36]$. Therefore we analyzed these homeobox proteins in GBM patients-derived orthotopic xenografts generated by V1G1 $1^{\text {Low }}$ or V1G1${ }_{\text {High }}$ NSand in human GBM tissues characterized for V1G1 expression. Overexpression of homeobox genes in $\mathrm{V} 1 \mathrm{G} 1^{\mathrm{HIGH}} \mathrm{NS}$ was also preserved in vivo (Fig. 1a and Fig. S1a). In fact, orthotopic brain tumors generated from V1G1 ${ }^{\mathrm{HIGH}} \mathrm{NS}$ contained more HOXA10- or POU3F2-positive cells ( $p=0.006$ and $p=0.0012$, respectively; Fig. $1 \mathrm{~b})$. Control brains from mice not injected with GBM NS were negative for all markers (Fig. S1b). These data confirm that upregulation of homeobox genes is a signature that defines V1G1-high gliomas.

\subsection{NS reprogram their microenvironment via large oncosomes loaded with V-ATPase V1G1 and homeobox proteins}

In the companion study (Terrasi et al., this issue) [36] in silico analysis of pathways associated to the V-ATPase-GBM-like phenotype identified cell-cell signaling, besides hox genes overexpression. This result, together with current knowledge regarding the importance of glioma 

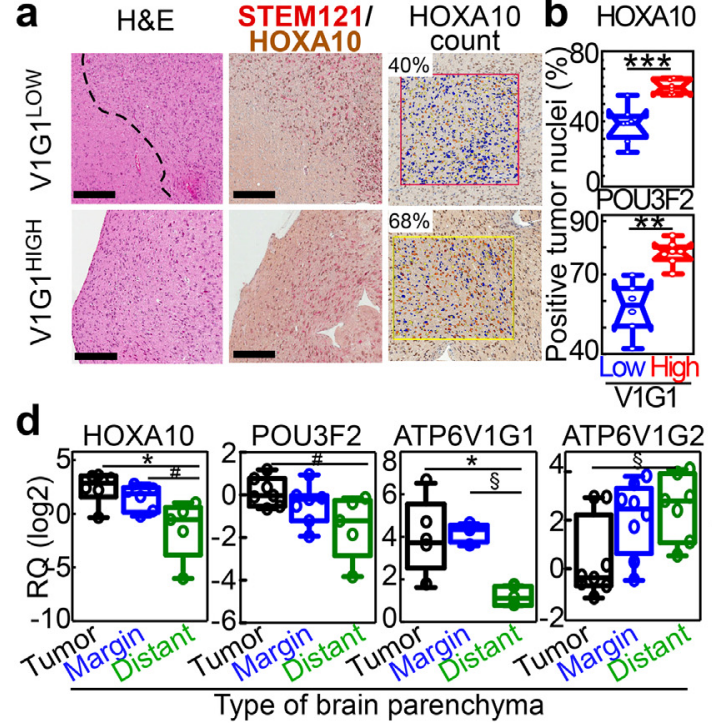

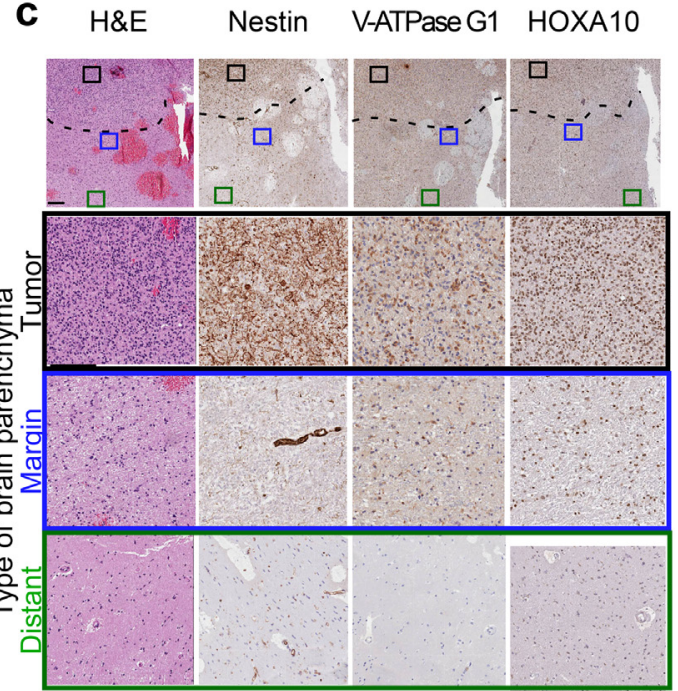

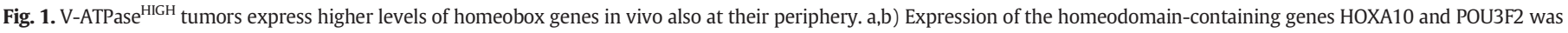

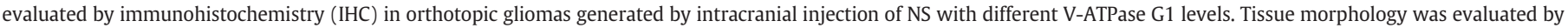

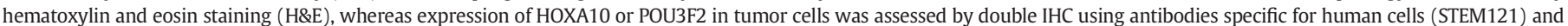

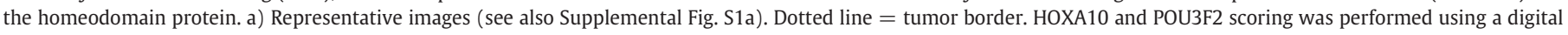

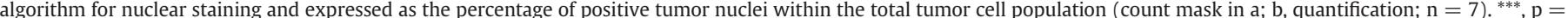

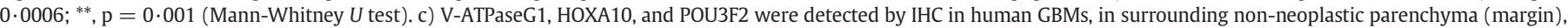

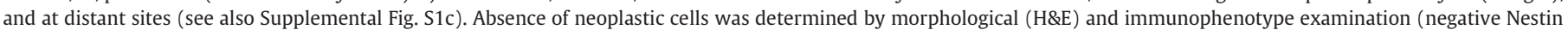

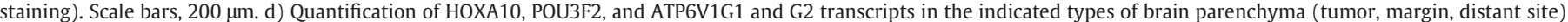

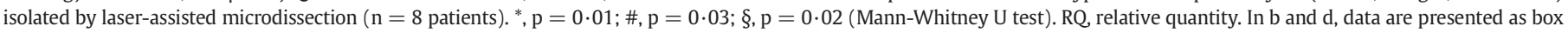
plots with whiskers indicating the minimal and maximal values. Each sample is a dot.

stem cells in influencing the non-neoplastic parenchyma, prompted us to examine expression of V-ATPase and homeobox proteins at tumor margins (defined as non-neoplastic areas in close proximity to the tumor), as well as at distant brain parenchyma sites, in a subset of GBM patients with elevated expression of V-ATPase G1 ( $n=11$; Fig. 1c and Fig. S1c). Tumor margins appeared significantly impacted by tumor proximity in that they displayed an intermediate level of V-ATPase and homeobox expression between that shown by glioma and normal (distant) brain tissues (Fig. 1c,d and Fig. S1c). We also evaluated Nestin, a marker of GBM cells, to verify that margins were devoid of tumor cells. Indeed, there was no difference in Nestin expression between the two types of non-neoplastic brain tissue (Fig. 1c and Fig. S1d).

Intermediate expression of V-ATPase and homeobox genes in nonneoplastic areas proximal to tumor suggests that GBM cells might deliver tumor-associated cargoes to nearby cells. Therefore, we analyzed whether GBM NS secrete EVs. Electron microscopy revealed that GBM NS generated and secreted a large number of EVs of different sizes (Fig. 2a and Fig. S2a). We focused our attention on large oncosomes (LO) because of their established role in delivering cargoes, including proteins, and their supposed tumor origins [7]. We isolated LO from NS culture medium (Fig. 2b) and assayed them for expression of specific protein markers (Fig. 2c) or for the presence of specific RNA (Fig. S2b). Next, we verified that purified LO from either NS V1G1 ${ }^{\text {Low }}$ or V1G1 $1^{\text {High }}$ were similarly internalized by recipient cells (Fig. 2d and Fig. S2c,d) of neoplastic or non-neoplastic (brain margins; Fig. S3a,b) histology to prove that they were functional. Then, we hypothesized that these vesicles were different in their contents with respect to V-ATPase G1 levels on the NS from which they originated. LO from V1G1 $1^{\mathrm{High}} \mathrm{NS}\left(\mathrm{LO}^{\mathrm{High}}\right)$ contained more homeobox transcripts than LO generated by V1G1 ${ }^{\text {Low }}$ NS (LO ${ }^{\text {Low }}$; Fig. 2e). Interestingly, $\mathrm{LO}^{\mathrm{High}}$ harbored higher amounts of V-ATPase G1 mRNA (Fig. 2e) and protein (Fig. 2f) than LO ${ }^{\text {Low }}$. Upon co-culture of $\mathrm{LO}^{\mathrm{High}}$ with recipient cells for 24 or $48 \mathrm{~h}$ (Fig. S2c), homeobox and V1G1 genes were overexpressed by recipient cells at the mRNA and protein level (Fig. 2g,h and Fig. S4a). This effect was not seen in cultures supplemented with $\mathrm{LO}^{\mathrm{Low}}$ (Fig. $2 \mathrm{~g}$ and Fig. S4a,b) and it was not due to lower content of LO from NS V1G1 $1^{\text {Low }}$ respect to NS V1G1 $1^{\text {High }}$ cultures (Fig. S4c,d). More interestingly, the molecular cargoes were expressed by recipient cultures up to 90 days from the LO supplementation (Fig. 2i).

From a functional point-of-view, supplementation of the culture medium with $\mathrm{LO}^{\mathrm{High}}$ increased the survival and proliferative activity of cultured non-neoplastic cells (derived from margin tissues; Fig. 3a-c and Fig. S5a,b) and glioma cells (LN229; Fig. S5c). When primary nonsphere-forming glioma cells were co-cultured with $\mathrm{LO}^{\mathrm{High}}$, they were induced to form NS, and their immunophenotype was altered as evidenced by decreased expression of GFAP protein (Fig. 3d). Finally, NS motility increased upon supplementation with $\mathrm{LO}^{\mathrm{High}}$ (Fig. 3e). Remarkably, a single supplementation with $\mathrm{LO}^{\mathrm{High}}$ was sufficient to induce primary glioma cultures to form long-lived NS (Fig. 3f). Both NS motility and clonogenicity was not affected by co-culture with $\mathrm{LO}^{\text {Low }}$ (Fig. S5d,e). The longevity of such NS correlated with HOX transcript levels measured on Day 6 from the start of co-culture (Fig. 3g); also, $\mathrm{LO}^{\text {High }}$ treated recipient cells upregulated expression of the HOXA10responsive gene CDKN1A (Fig. S5f) [19], suggesting that these effects were related to transfer of HOX-genes.

Given this functional reprogramming, we examined the transcriptome of primary non-sphere-forming glioma cells co-cultured for 6 days with empty medium (mock) or with medium containing $\mathrm{LO}^{\text {High }}$. Gene set enrichment analysis (GSEA) revealed that LO-supplemented cultures activated a number of cancer-related pathways, including those involved in glioma V-ATPase-related signaling (Notch, mTOR, and lysosomal transport) [20], as well as cell proliferation and cell transcription programs (Fig. 3h and Table S1). Finally, 10 among the top genes identified in the transcriptomic analysis as differentially upregulated in LO treated samples (FC $>2, p<0.01$ by Wilcoxon matched paires test; Table S2) were analyzed by qPCR in five cultures of non-sphereforming glioma cells (Fig. S5 g). We could validate overexpression of 
a

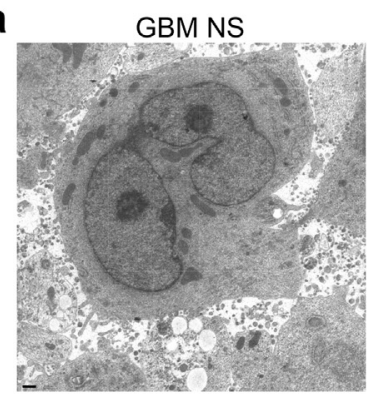

d

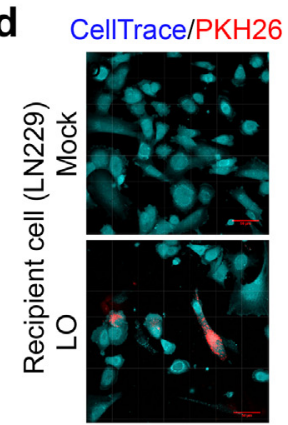

g
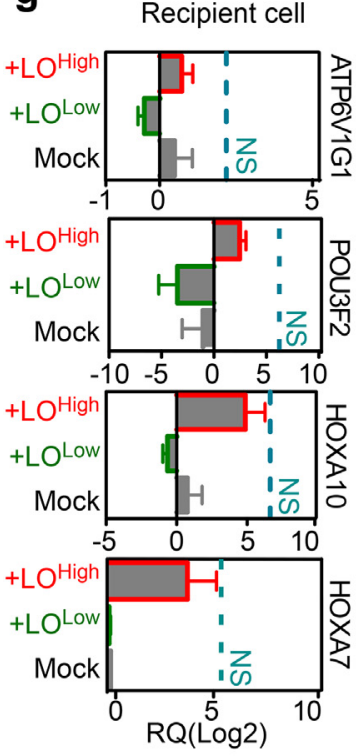

b

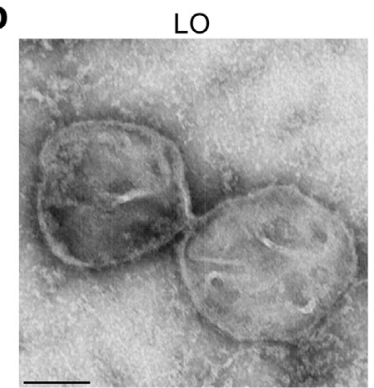

C MW GBM (KD) LO NS

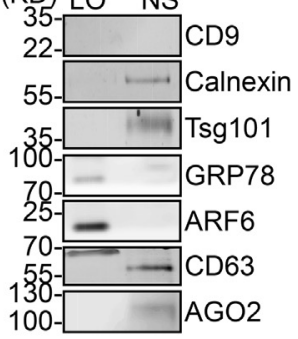

$\mathbf{f}$

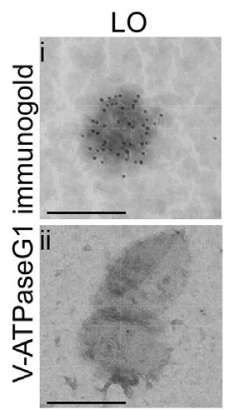

i h
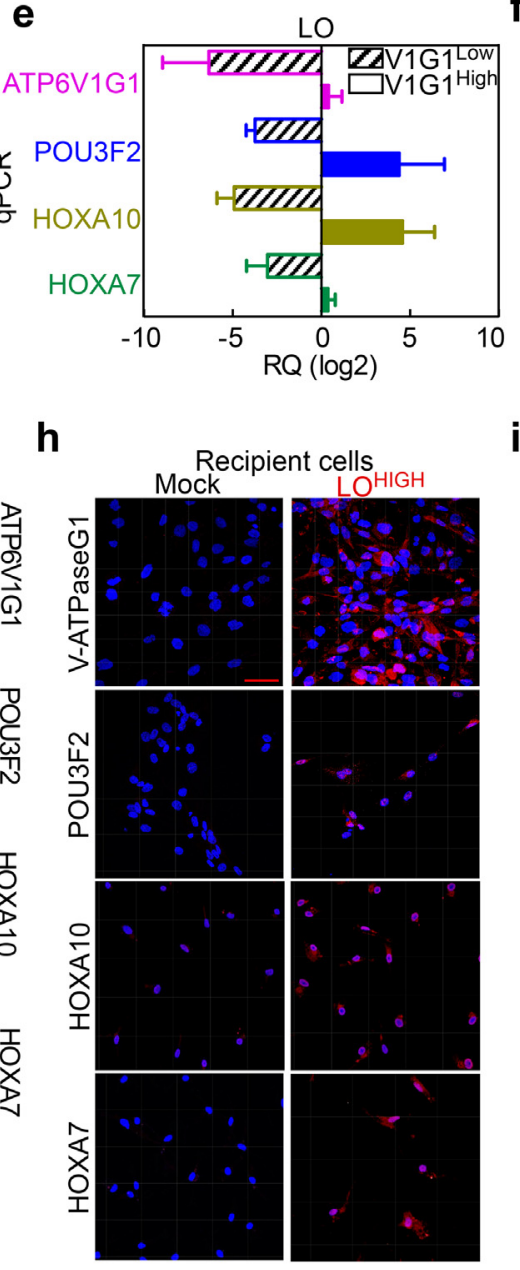

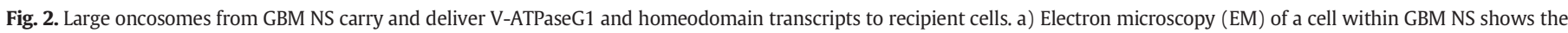

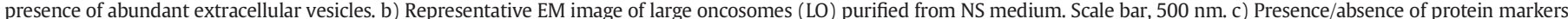

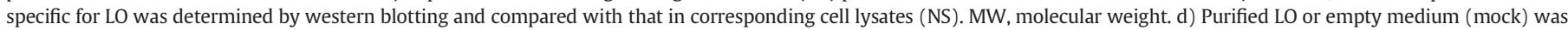

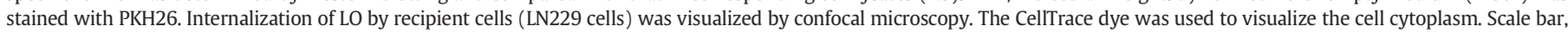

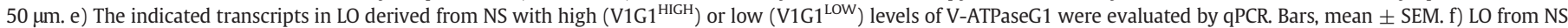

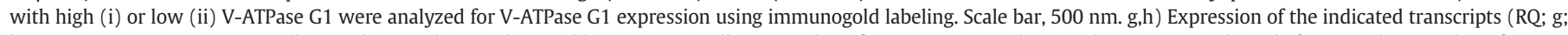

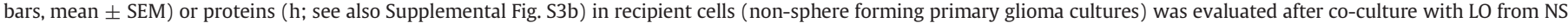

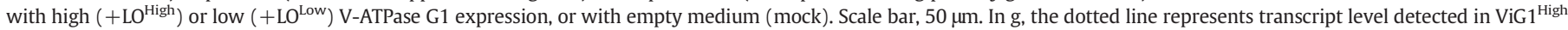

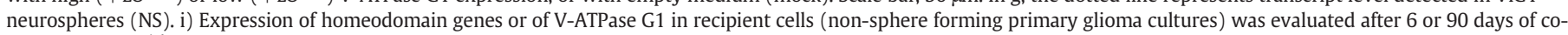
culture with $\mathrm{LO}^{\mathrm{High}}$. $\mathrm{RQ}$, relative quantity. Bars, mean $\pm \mathrm{SEM}$.

EGFR, a known GBM key gene and represented cargo of glioma EVs [8], and of transcripts involved in glioma tumorigenesis or drug resistance such as TTLL7 [21] and RFC5 [22]. Furthermore we confirmed upregulation in LO-supplemented cultures of genes involved in cancer cell proliferation, such as BOC[23] and invasion, such as TCTN2 [24] or NFIB [25]. This experiment lends further support to the hypothesis that LO derived from $\mathrm{V} 1 \mathrm{G} 1^{\mathrm{High}} \mathrm{NS}$ induce extensive transcriptional changes in recipient cells, thereby promoting tumor growth.

\subsection{LO derived from GBM patients carry V-ATPaseG1 and homeobox proteins}

Next, we asked whether LO were also present in the circulation of glioma patients before surgery, and whether their molecular content reflected our observations regarding GBM NS. Not only were LO isolated from the serum of glioma patients $(n=28$; Fig. $4 a)$, they were also functional (i.e., were internalized by recipient cells; 
a

Recipient cells:

d

Recipient cells:

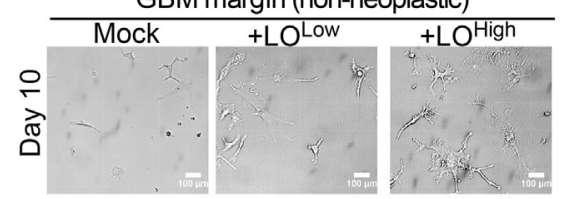
primary glioma cultures (non-sphere forming)

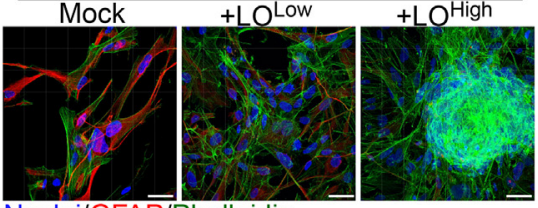

b
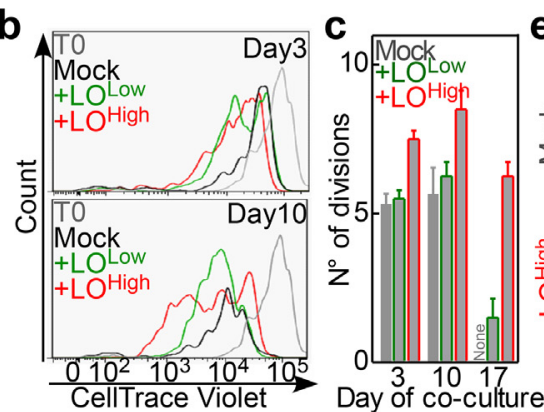

e Nuclei/GFAP/Phalloidin

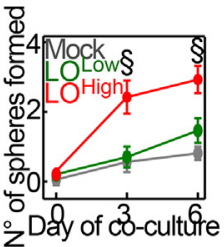

f
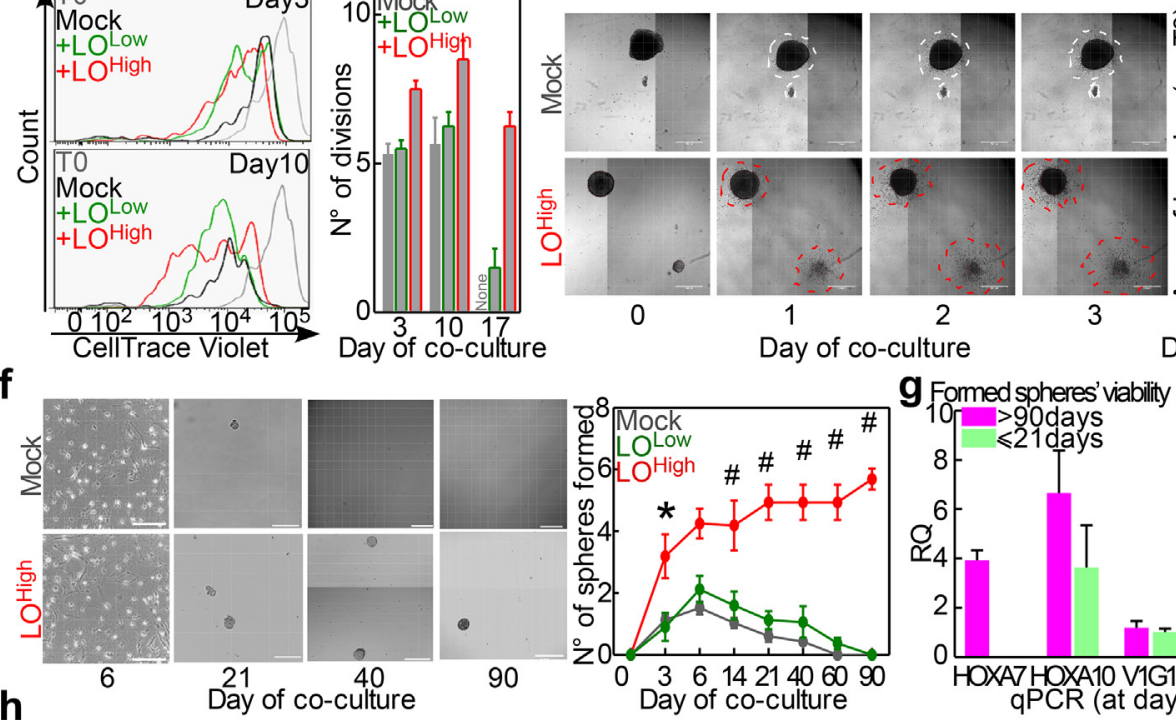

Day of co-culture
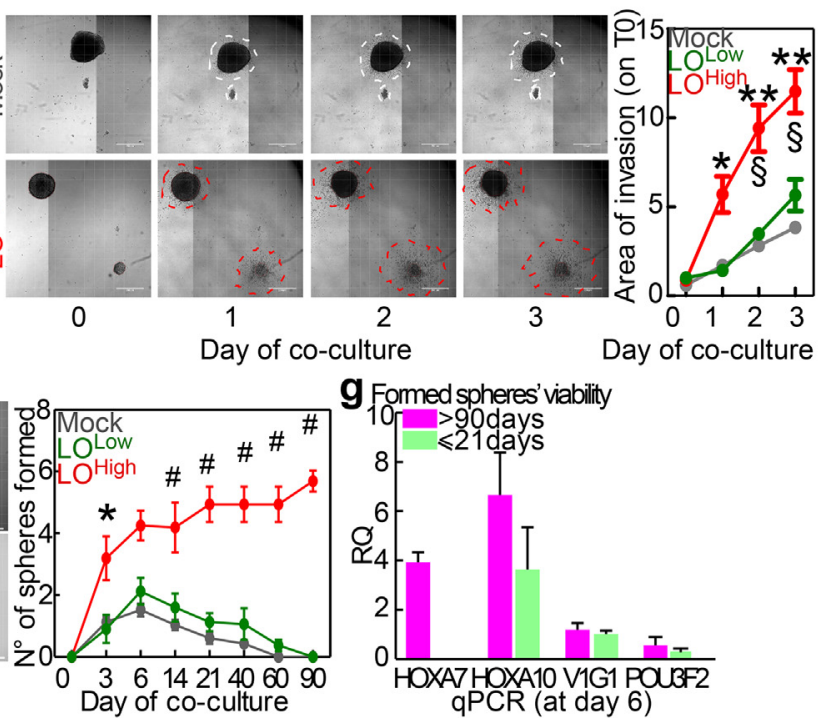

9 Formed spheres' viability
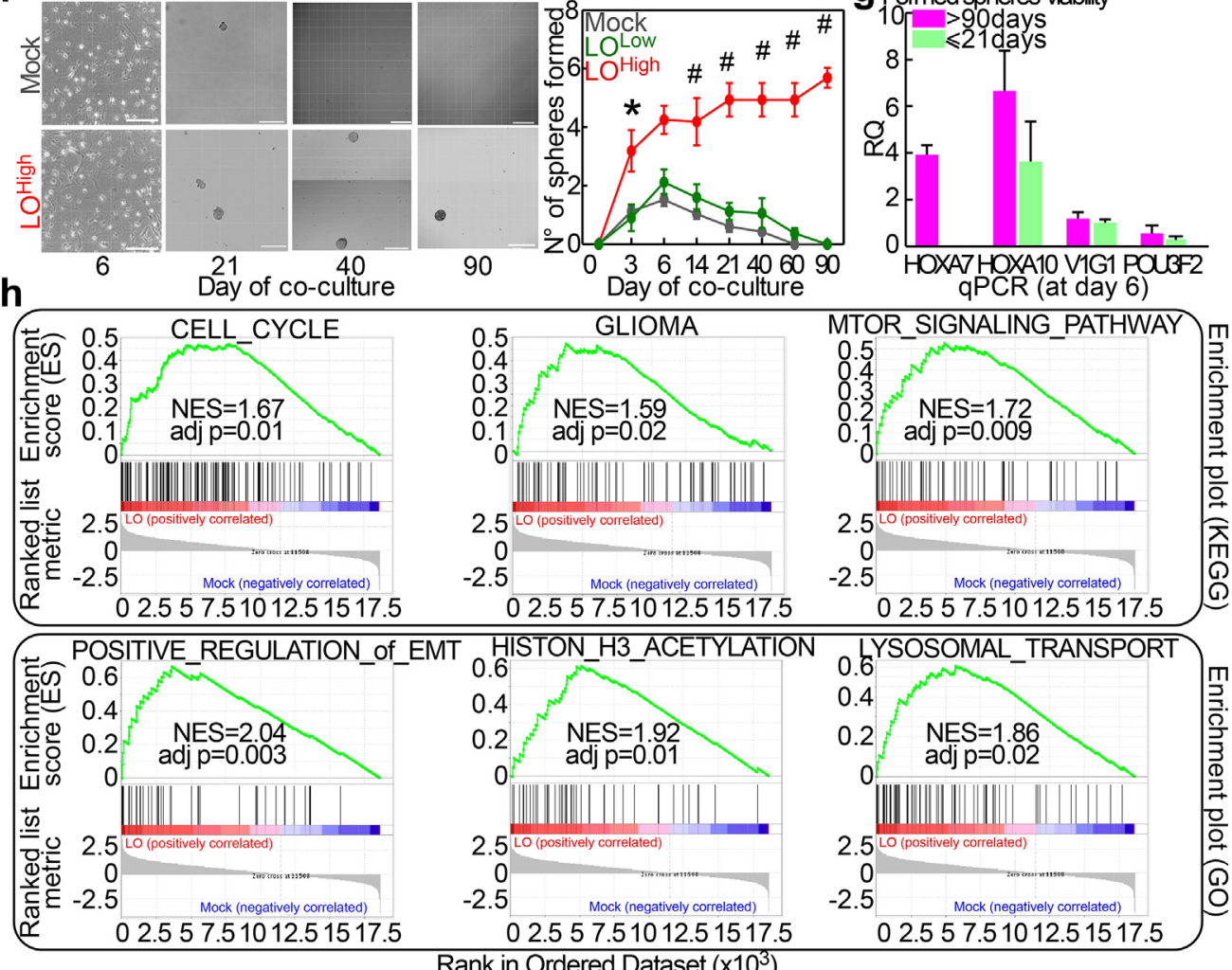

Rank in Ordered Dataset $\left(x 10^{3}\right)$

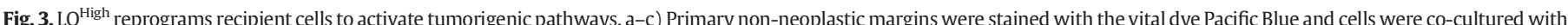

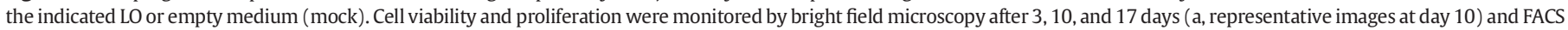

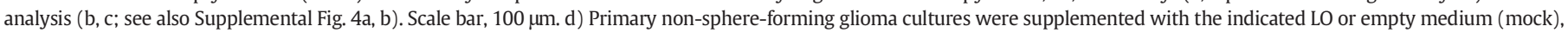

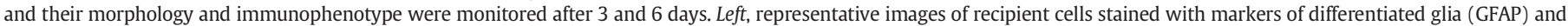

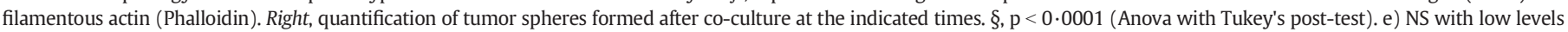

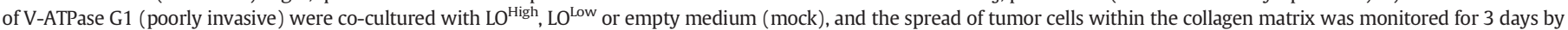

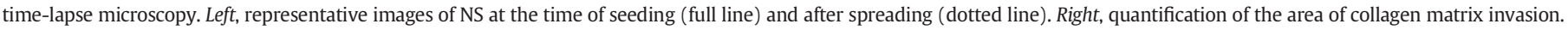

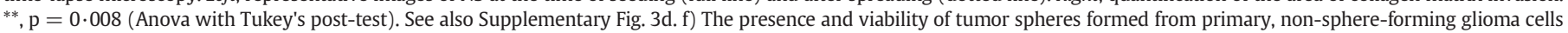

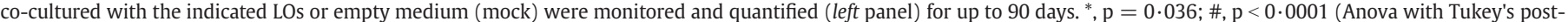

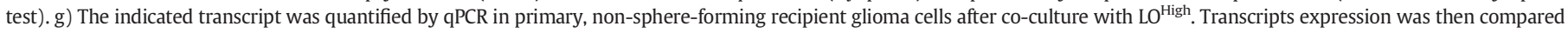

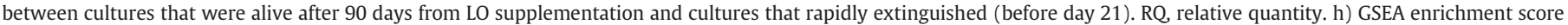

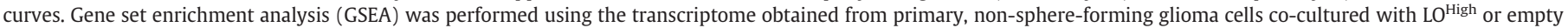

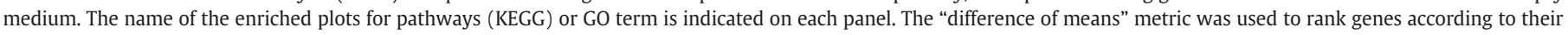

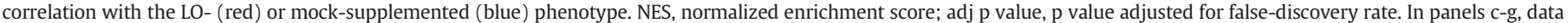
are presented as mean \pm SEM. (For interpretation of the references to colour in this figure legend, the reader is referred to the web version of this article.) 
a

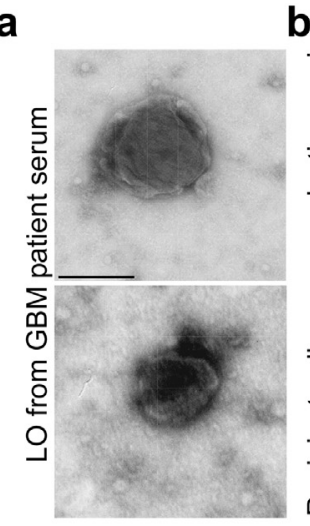

d

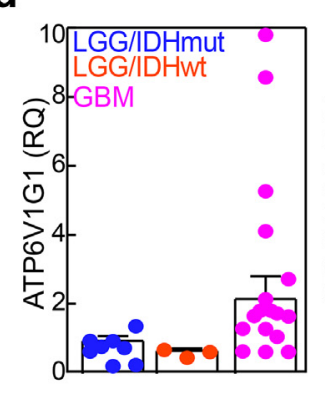

$\mathbf{f}_{\mathrm{p}}$

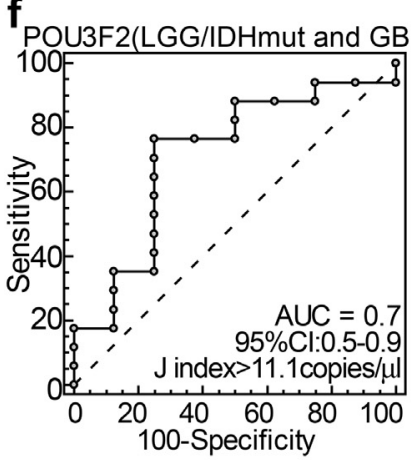

b
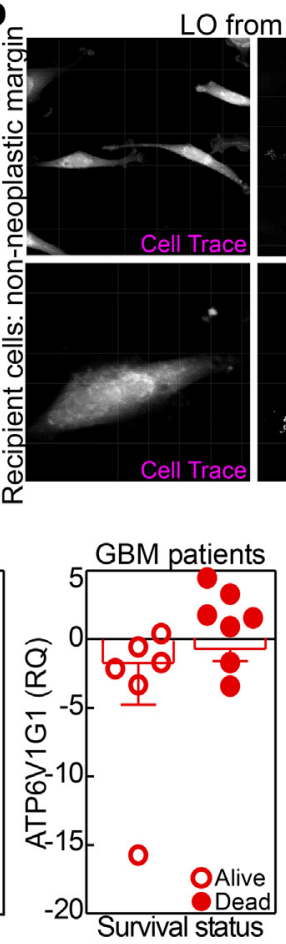

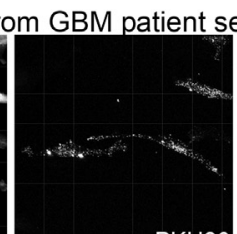

PKH26

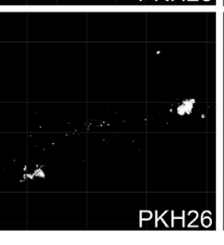

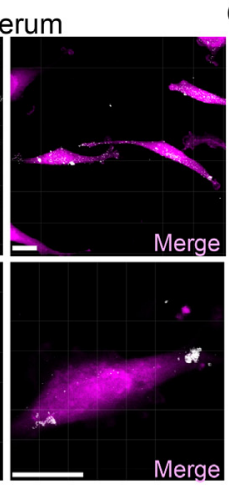

C $_{\text {MW }}$ Patient ID:

(KD) $133 \quad 92 \quad 104$
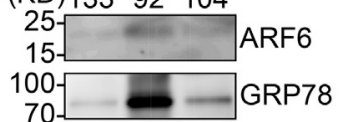

HOXA10
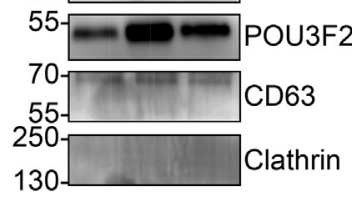

e POU3F2

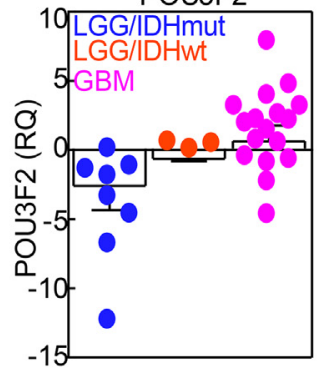

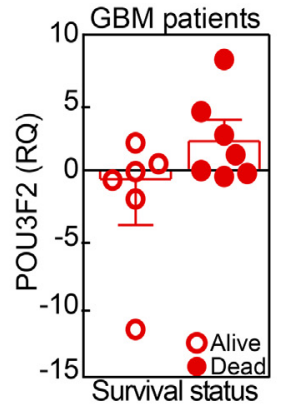

h
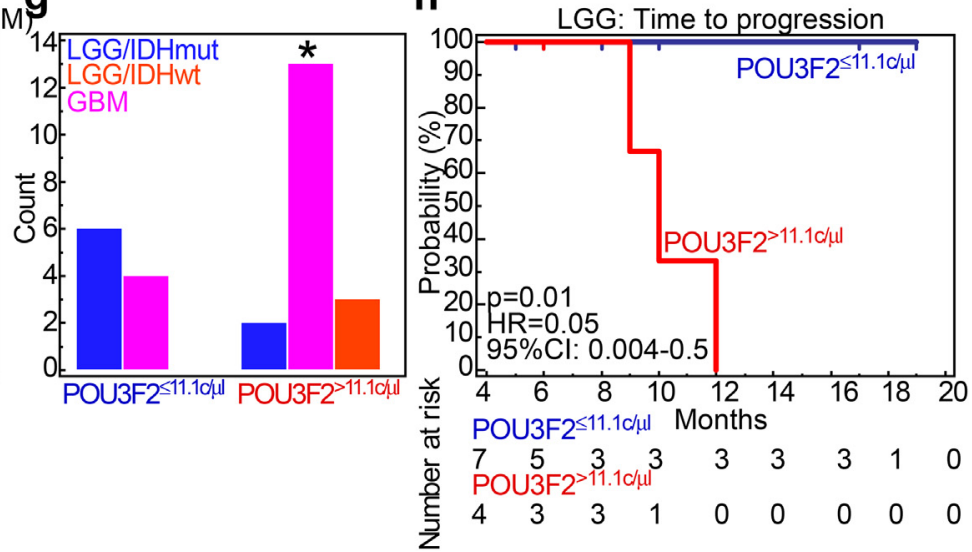

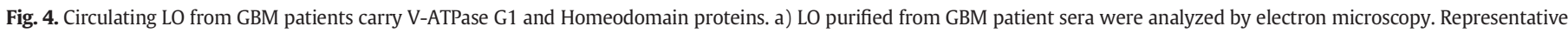

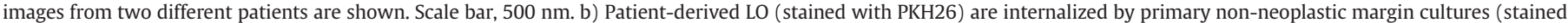

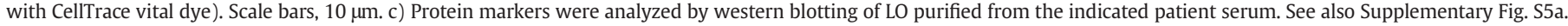

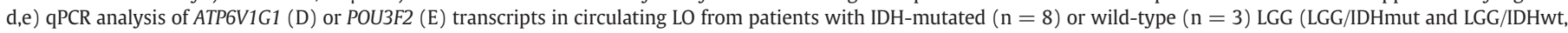

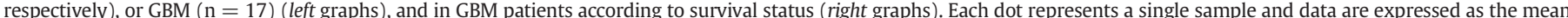

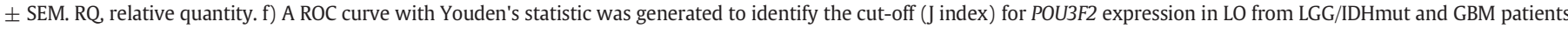

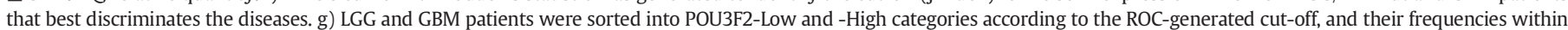

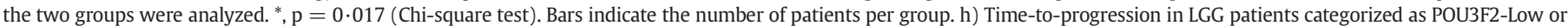
High was analyzed using Kaplan-Meier curves and the Log-Rank test.

Fig. 4b) and they expressed specific markers (CD63, ARF6, and GRP78; Fig. 4c; Fig. S6a) and homeobox genes (Fig. 4c).

Regarding their molecular cargoes, ATP6V1G1 transcript was higher in GBM patients-derived LO than in LGG, and was elevated in GBM patients who died of disease during follow-up (mean survival time: 10.6 months; Fig. 4d). Expression of POU3F2 mRNA was higher in LGG/IDHwt than in LGG/IDHmut, as well as being upregulated in GBM. POU3F2 was also high among GBM patients who died of disease (Fig. 4e). To confirm the qPCR data and to translate those observations into clinically meaningful markers, we measured expression of POU3F2 in patient-derived LO using digital PCR (Supplemental Fig. S6b). Interestingly, the value of POU3F2 expression that best discriminated LGG/IDHmut from GBM cases (corresponding to 11.1 copies/ $\mu \mathrm{l}$ ) (Fig. 4f) classified LGG/IDHwt cases alongside GBM (Fig. 4g) and it was also predictive of shorter disease-free survival time in LGG patients ( $p=0.01$; Fig. $4 \mathrm{~h}$ ). These data, though preliminary, show that aggressive gliomas actively secrete LO loaded with V1G1 and POU3F2 oncogenic cargoes into the circulation, suggesting the possibility of using these markers for staging or follow-up purposes.

\subsection{The molecular content and biological activity of LO are sensitive to impairment of $V$-ATPase pumps in NS}

Finally, to provide a preliminary rational for therapeutic intervention, we asked whether the molecular content and functional effects of LO were modulated by impairment of V-ATPase in NS.

Treatment with the V-ATPase inhibitor Bafilomycin A1 (BafA1; Fig. 5a), or transient silencing of V1G1 by siRNA (Fig. 5b), decreased 


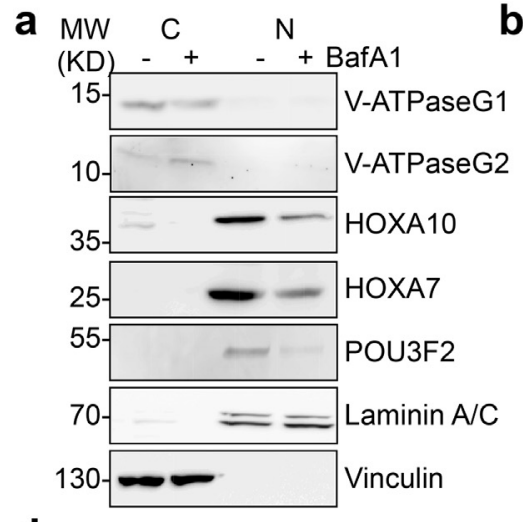

d
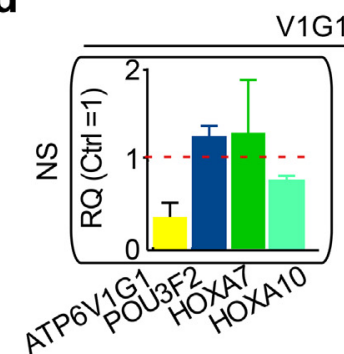

b

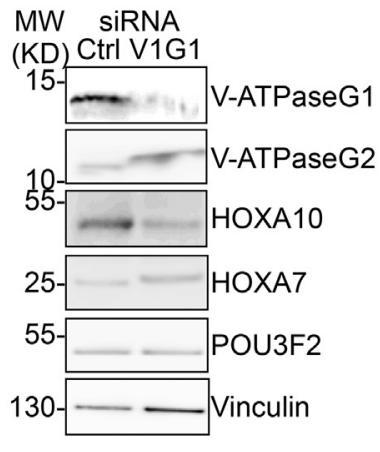

C

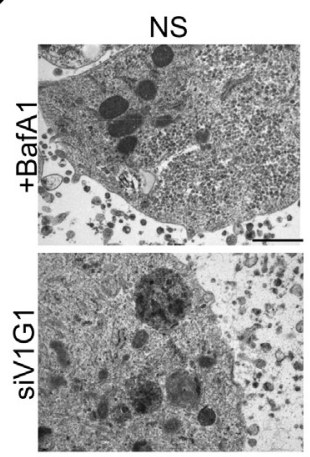

f

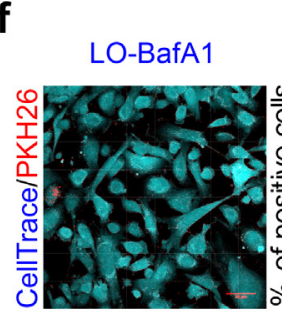

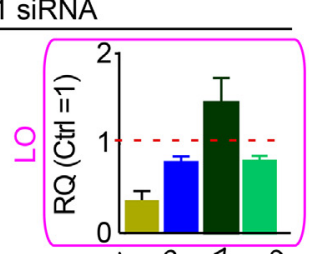

स18 18080 e

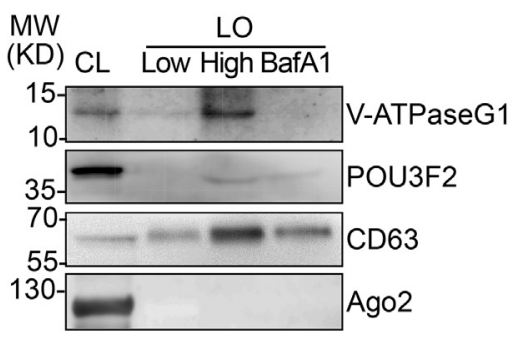

g Recipient cells:non-neoplastic margin $\mathbf{h}$
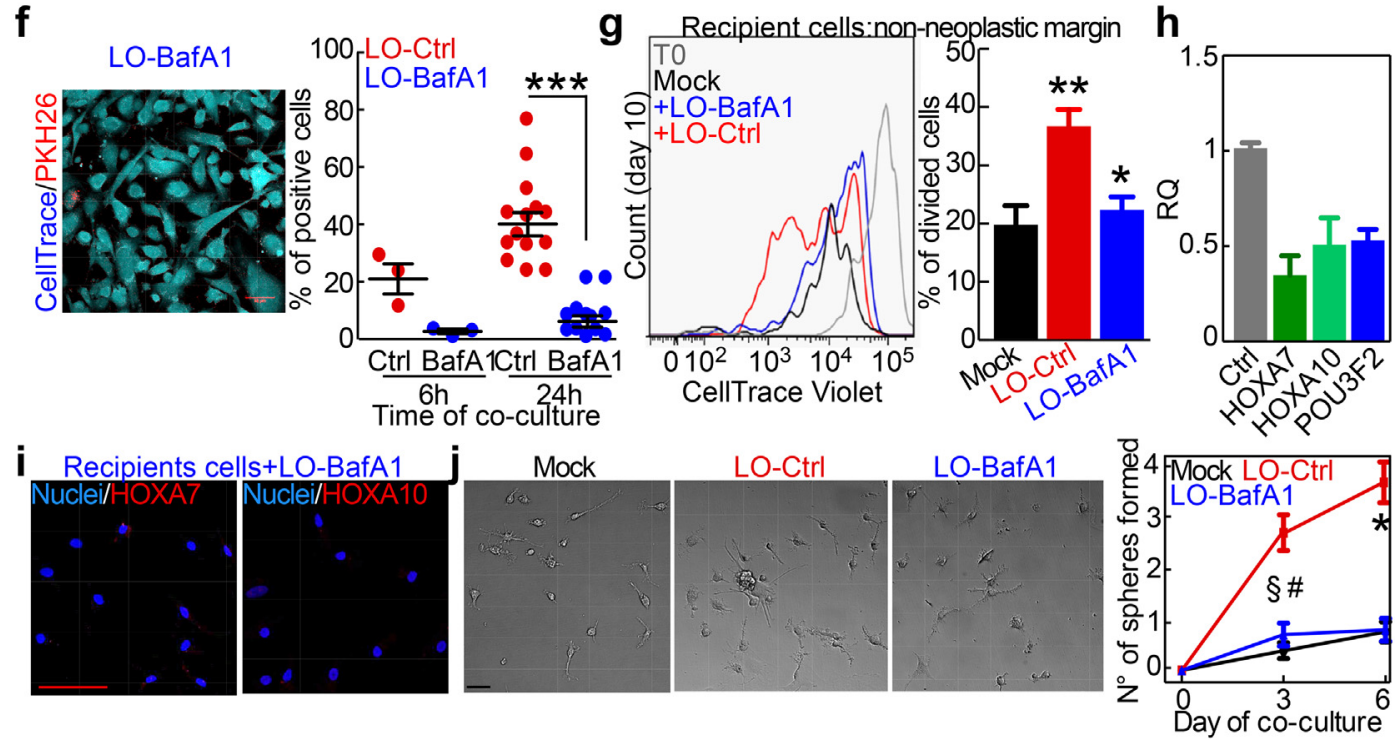

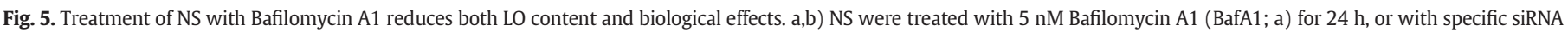

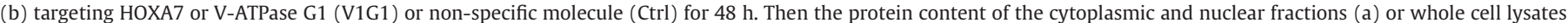

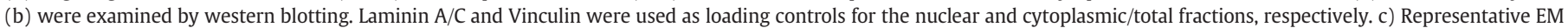

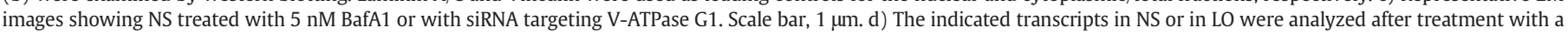

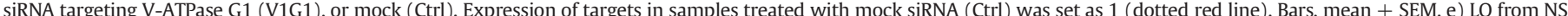

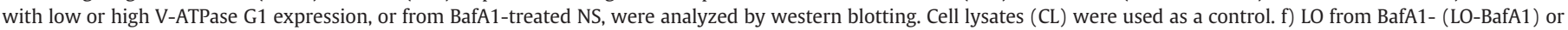

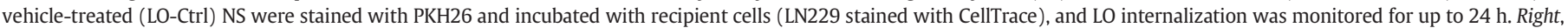

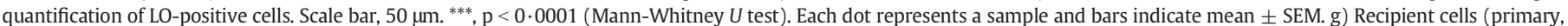

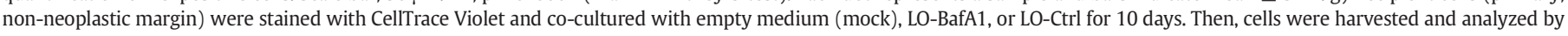

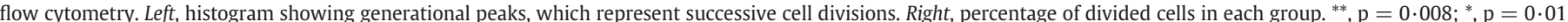

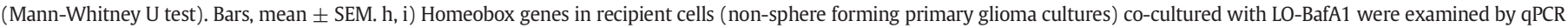

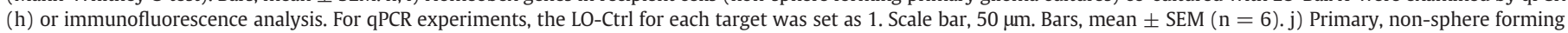

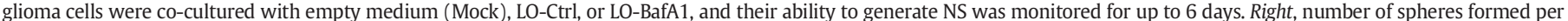

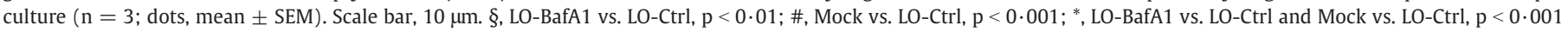
(Kruskal-Wallis test with Dunn's post-test). (For interpretation of the references to colour in this figure legend, the reader is referred to the web version of this article.)

expression of homeobox proteins in GBM NS and led to V1G2 upregulation (Fig. 5a,b). Next, we analyzed whether these treatments altered LO formation, molecular content, and biological function. LO production by NS was not affected by either BafA1 treatment or V1G1 knockdown (Fig. 5c and S4c,d). Besides reducing transcript levels in NS, silencing of V1G1 decreased its messenger RNA together with POU3F2 and
HOXA10 mRNAs in NS and LO (Fig. 5d). These data were confirmed at the protein level (Fig. 5e). From a functional point-of-view, uptake of LO from BafA1-treated NS (LO-BafA1) by recipient cells was less effective than that by control cells (Fig. 5f). Moreover, LO-BafA1 were not able to promote cell growth in non-neoplastic margins (Fig. 5g) and did not upregulate homeobox transcripts in recipient cells (Fig. 5h,i). 
Finally, addition of LO-BafA1 to primary glioma cultures had no effect on sphere formation (Fig. 5j).

To test whether the observed effects of V-ATPase impairment on expression of homeobox genes were specific for V-ATPase activity or related to lysosomal dysfunction, we treated LO-forming NS with ammonium chloride ( $\mathrm{NH} 4 \mathrm{Cl}$ ), which neutralizes lysosomal $\mathrm{pH}$. Exposure of NS to $50 \mathrm{mM} \mathrm{NH4Cl}$ reduced intracellular and lysosomal acidification in a manner similar to BafA1 (Fig. S7a,b). Interestingly, $\mathrm{NH} 4 \mathrm{Cl}$ partially reduced the clonogenicity of primary GBM cultures and NS invasiveness (Fig. S7c and d, respectively) without affecting NS viability (Fig. S7e). However, treatment of NS with $\mathrm{NH} 4 \mathrm{Cl}$ did not alter expression of homeobox genes (Fig. S7f). These results suggest that V-ATPase activity, rather than lysosomal function, is responsible for cell-cell signaling in GBM stem cells.

\section{Discussion}

In this study we show that highly tumorigenic glioma stem cells secrete in their microenvironment large oncosomes, a type of tumorspecific EVs loaded with the homeobox genes POU3F2 and HOXA10, and with the V-ATPase subunit G1. LOs are transferred to both neoplastic and non-neoplastic recipient cells, thus mediating their reprogramming toward an oncogenic state activating proliferative and motility pathways.

Remarkably, LOs loaded with V1G1 and POU3F2 were present in the circulation of glioma patients, and their levels increased with tumor grade or in patients with a poorer outcome. Based on elevated POU3F2 levels in LO of glioma patients, we were able to sort out IDHwt lower-grade glioma and GBM from IDHmut cases, and we found that the same cut-off is also prognostic for lower-grade glioma patients in term of disease progression.

Finally, impairment of V-ATPase activity in NS had a marked effect on LO content and abolished their oncogenic effect on recipient cells. This suggests that the pump is required for delivery of oncogenic signals, carried by LO, to the tumor microenvironment. Besides providing novel insights in glioma progression, our study provides preliminary data for potential non-invasive biomarkers useful for stratification and follow-up of LGG patients.

In a companion study (Terrasi et al., this issue) [36] we show that alternative expression of V-ATPase subunits stratifies the heterogeneous class of LGG/IDHwt and identifies the cases with GBM-like prognosis. Interestingly, the GBM-like pump signature, defined as over-expression of subunit V1G1 over V1G2, of V0A2 over V0A1 and down-regulation of $\mathrm{V} 1 \mathrm{C} 1$, associates with the hox genes and with cell-cell communication signatures.

Using primary neurospheres cultures we confirmed that Hox genes are more expressed in cells with the GBM-like pump (Terrasi et al., this issue) [36]. Here we extend this information showing that V-ATPase G1, HOXA10 and POU3F2 are found in vivo in the tumor surrounding milieu of GBM. The proposed mechanism is the delivery of these factors through large oncosomes shed from GBM stem-like cells to the microenvironment. In support of this, we show that V-ATPase G1 protein is present in LO secreted by GBM-NS and that homeodomain proteins and V1G1 are present also in LO found in the circulation of glioma patients.

EVs play important roles in brain tumors. In glioma, EVs were firstly described as transporters of the oncogene EGFRvIII molecule [8].

Besides mediating cell-cell communications by carrying signaling molecules and nucleic acids, EVs recapture intratumor heterogeneity and glioma stem cells hierarchy. Furthermore, EVs are proposed to be partly responsible for the molecular plasticity exhibited by glioma stem cells, as well as to participate in the switching from a transcriptomic subtype to another [11].

Among EVs, LO have been previously described in metastatic prostate cancer [6], in which they are internalized by tumor cells as well as by the surrounding stroma. Consistent to our results, LO were also found in the circulation of prostate cancer patients and their abundance predicted disease progression.

Monitoring of disease progression and therapeutic responses after resection in GBM patients is currently achieved by serial neurological examination and MRI analyses. This procedure avoids multiple brain biopsies that are highly invasive and impractical in the routine. Therefore, detection of recurrence is limited by the resolution of the MRI (about 2-3 mm). Moreover, therapeutic protocols for GBM patients are based on radiation with concurrent chemotherapy [26]. This often results in a phenomenon called pseudoprogression, which mimics real tumor progression on MRI [27] and makes disease monitoring more time consuming.

In light of such limitations, there is a clear clinical need for novel diagnostic tools that would allow timely and accurate detection of recurrence. Together, our and previous data on EVs detection as a way to predict disease status, support the use of LO as a potential circulating biomarker also in glioma patients. Indeed, LO were described to be characteristic of metastatic cancer cells that are though to drive tumor recurrence [6,7]. Moreover, LO should be more accurate in tumor monitoring than EVs because there are mainly released by neoplastic cells [7,8], although it has been reported that large EVs were also detected in the supernatant of normal dendritic cells [28]. Therefore the presence and detection of specific tumor-marker in circulating LO rather than standard EV markers is likely to be key in determining whether they originate from the cancer compartment [29].

Previous reports showed that V-ATPase could govern cell-cell and tumor microenvironment communication [30,31]. Moreover, a role for V-ATPase in regulating EVs production and uptake has been documented in both non-mammalian [32] and mammalian organisms. VATPase subunits V1B1 and B2 have been described in urinary EVs in human distal renal tubular acidosis disease [33], whereas V-ATPase V1E1 was detected in EVs of metastatic cells of different origins [34].

Our data are in line with these reports and expand current knowledge about V-ATPase involvement in human cancers, by strengthening the connection between specific expression of an oncogenic pump and presence of cancer stem-like cells [12,13,35]. From a clinical stand-point, such signature is a predictor of an aggressive disease and of short progression-free survival.

Our studies collectively highlight a strong connection between V-ATPase and stem cell activity in cancer (Terrasi et al., this issue) $[13,36]$. We envisage that GSC might select specific V-ATPase and Hox factors delivered by LOs to reprogram non-cell-autonomously their microenvironment toward a pro-tumorigenic state. Our novel understanding of how tumor cells have learned to influence and instruct the non-neoplastic milieu will be crucial to develop future therapeutic attempt to block tumor invasion, as well as to overcome resistance to therapy. Overall, this study paves the way toward the use of GBM LOs as a treatment targets as well as diagnostic markers.

\section{Author contributions statement}

I.B. and A.T. conceived and designed the experiments and prepared the tables/figures; I.B. conducted in vitro experiments, vesicles isolation and characterization; A.T. performed statistical analyses; C.M., M.F. and L.O. performed in vivo experiments and analyzed the data; G.G. and A.M.S. performed qPCR, in vitro experiments and analyzed data; A.D.C., M.C. provided patients' samples and clinical data; P.B. performed EM experiments; S.F. performed IHC experiments and analyzed the data; K.T. performed microarray analysis and analyzed the data; T.V. and V.V. drafted and wrote the manuscript; All authors reviewed the manuscript.

\section{Declaration of interests}

The authors declare no competing interests. 


\section{Acknowledgments}

This work was supported by Fondazione Cariplo (2014-1148 to VV), Fondazione IRCCS Ca' Granda, and Fondazione INGM Grant in Molecular Medicine 2014 (to VV) and by the Ricerca Corrente program 2017 (to SF). AMS and MF were supported by a Fellowship from the Doctorate School in Molecular and Translational Medicine of Milan University. We are thankful to dr. Mariacarla Panzeri from ALEMBIC-Advanced Light and Electron Microscopy Biolmaging Center (San Raffaele Scientific Institute) for technical help with electron microscopy, to prof. Valentina Bollati from the Department of Clinical Sciences and Community Health (University of Milan) for help with digital PCR and NTA experiments, and to prof. Nino Neri from the Department of Oncology and Hemato-Oncology (University of Milan), for technical help with microarray analysis. The authors are thankful to the INGM Imaging Facility for scientific and technical assistance.

\section{Appendix A. Supplementary data}

Supplementary data to this article can be found online at https://doi. org/10.1016/j.ebiom.2019.01.051.

\section{References}

[1] Hochberg FH, Atai NA, Gonda D, et al. Glioma diagnostics and biomarkers: an ongoing challenge in the field of medicine and science. Expert Rev Mol Diagn 2014;14(4): 439-52.

[2] Hanahan D, Weinberg RA. Hallmarks of cancer: the next generation. Cell 2011;144 (5):646-74.

[3] Zappulli V, Friis KP, Fitzpatrick Z, Maguire CA, Breakefield XO. Extracellular vesicles and intercellular communication within the nervous system. J Clin Invest 2016; 126(4):1198-207.

[4] Al-Nedawi K, Meehan B, Micallef J, et al. Intercellular transfer of the oncogenic receptor EGFRvIII by microvesicles derived from tumour cells. Nat Cell Biol 2008;10 (5):619-24.

[5] Peinado H, Aleckovic M, Lavotshkin S, et al. Melanoma exosomes educate bone marrow progenitor cells toward a pro-metastatic phenotype through MET. Nat Med 2012;18(6):883-91.

[6] Di Vizio D, Morello M, Dudley AC, et al. Large oncosomes in human prostate cancer tissues and in the circulation of mice with metastatic disease. Am J Pathol 2012;181 (5):1573-84.

[7] Minciacchi VR, You S, Spinelli C, et al. Large oncosomes contain distinct protein cargo and represent a separate functional class of tumor-derived extracellular vesicles. Oncotarget 2015;6(13):11327-41.

[8] Skog J, Wurdinger T, van Rijn S, et al. Glioblastoma microvesicles transport RNA and proteins that promote tumour growth and provide diagnostic biomarkers. Nat Cell Biol 2008;10(12):1470-6.

[9] Kucharzewska P, Christianson HC, Welch JE, et al. Exosomes reflect the hypoxic status of glioma cells and mediate hypoxia-dependent activation of vascular cells during tumor development. Proc Natl Acad Sci U S A 2013;110(18):7312-7.

[10] Westphal M, Lamszus K. Circulating biomarkers for gliomas. Nat Rev Neurol 2015;11 (10):556-66.

[11] Nakano I, Garnier D, Minata M, Rak J. Extracellular vesicles in the biology of brain tumour stem cells-Implications for inter-cellular communication, therapy and biomarker development. Semin Cell Dev Biol 2015;40:17-26.

[12] Stransky L, Cotter K, Forgac M. The function of V-ATPases in cancer. Physiol Rev 2016;96(3):1071-91.
[13] Di Cristofori A, Ferrero S, Bertolini I, et al. The vacuolar H+ ATPase is a novel therapeutic target for glioblastoma. Oncotarget 2015;6(19):17514-31.

[14] Louis DN, Perry A, Reifenberger G, et al. The 2016 World Health Organization classification of tumors of the central nervous system: a summary. Acta Neuropathol 2016:131(6):803-20.

[15] Valtorta S, Lo Dico A, Raccagni I, et al. Metformin and temozolomide, a synergic option to overcome resistance in glioblastoma multiforme models. Oncotarget 2017;8 (68):113090-104.

[16] Minciacchi VR, Spinelli C, Reis-Sobreiro M, et al. MYC mediates large oncosomeinduced fibroblast reprogramming in prostate cancer. Cancer Res 2017;77(9): 2306-17.

[17] Lobb RJ, Becker M, Wen SW, et al. Optimized exosome isolation protocol for cell culture supernatant and human plasma. J Extracell Vesicles 2015;4:27031.

[18] Ria R, Todoerti K, Berardi S, et al. Gene expression profiling of bone marrow endothelial cells in patients with multiple myeloma. Clin Cancer Res 2009;15(17): 5369-78.

[19] Bromleigh VC. Freedman LP. p21 is a transcriptional target of HOXA10 in differentiating myelomonocytic cells. Genes Dev 2000;14(20):2581-6.

[20] Cotter K, Stransky L, McGuire C, Forgac M. Recent insights into the structure, regulation, and function of the V-ATPases. Trends Biochem Sci 2015;40(10):611-22.

[21] Katsetos CD, Reginato MJ, Baas PW, et al. Emerging microtubule targets in glioma therapy. Semin Pediatr Neurol 2015;22(1):49-72.

[22] Peng WX, Han X, Zhang CL, et al. FoxM1-mediated RFC5 expression promotes temozolomide resistance. Cell Biol Toxicol 2017;33(6):527-37.

[23] Mille F, Tamayo-Orrego L, Levesque M, et al. The Shh receptor Boc promotes progression of early medulloblastoma to advanced tumors. Dev Cell 2014;31(1):34-47.

[24] Cano-Rodriguez D, Campagnoli S, Grandi A, et al. TCTN2: a novel tumor marker with oncogenic properties. Oncotarget 2017;8(56):95256-69.

[25] Denny SK, Yang D, Chuang CH, et al. Nfib promotes metastasis through a widespread increase in chromatin accessibility. Cell 2016;166(2):328-42.

[26] Hygino da Cruz Jr LC, Rodriguez I, Domingues RC, Gasparetto EL, Sorensen AG. Pseudoprogression and pseudoresponse: imaging challenges in the assessment of posttreatment glioma. AJNR Am J Neuroradiol 2011;32(11):1978-85.

[27] Santiago-Dieppa DR, Steinberg J, Gonda D, Cheung VJ, Carter BS, Chen CC. Extracellular vesicles as a platform for 'liquid biopsy' in glioblastoma patients. Expert Rev Mol Diagn 2014;14(7):819-25.

[28] Kowal J, Arras G, Colombo M, et al. Proteomic comparison defines novel markers to characterize heterogeneous populations of extracellular vesicle subtypes. Proc Natl Acad Sci U S A 2016;113(8):E968-77.

[29] Meehan B, Rak J, Di Vizio D. Oncosomes - large and small: what are they, where they came from? J Extracell Vesicles 2016;5:33109.

[30] Michel V, Licon-Munoz Y, Trujillo K, Bisoffi M, Parra KJ. Inhibitors of vacuolar ATPase proton pumps inhibit human prostate cancer cell invasion and prostate-specific antigen expression and secretion. Int J Cancer 2013;132(2):E1-10.

[31] Wang D, Epstein D, Khalaf O, et al. Ca2+-Calmodulin regulates SNARE assembly and spontaneous neurotransmitter release via v-ATPase subunit V0a1. J Cell Biol 2014; 205(1):21-31.

[32] Liegeois S, Benedetto A, Garnier JM, Schwab Y, Labouesse M. The V0-ATPase mediates apical secretion of exosomes containing Hedgehog-related proteins in Caenorhabditis elegans. J Cell Biol 2006;173(6):949-61.

[33] Pathare G, Dhayat NA, Mohebbi N, et al. Changes in V-ATPase subunits of human urinary exosomes reflect the renal response to acute acid/alkali loading and the defects in distal renal tubular acidosis. Kidney Int 2018;93(4):871-80.

[34] Guo H, Chitiprolu M, Roncevic L, et al. Atg5 disassociates the V1V0-ATPase to promote exosome production and tumor metastasis independent of canonical macroautophagy. Dev Cell 2017;43(6) [716-30 e7].

[35] Salerno M, Avnet S, Bonuccelli G, Hosogi S, Granchi D, Baldini N. Impairment of lysosomal activity as a therapeutic modality targeting cancer stem cells of embryonal rhabdomyosarcoma cell line RD. PLoS One 2014;9(10):e110340.

[36] Terrasi A, Bertolini I, Martelli C, et al. Specific V-ATPase expression sub-classifies IDHwt lower-grade gliomas and impacts glioma growth in vivo. EBioMedicine: https://www.ebiomedicine.com/article/S2352-3964(19)30058-1/fulltext 